\title{
Necessary and Sufficient Conditions for Difference Flatness
}

\author{
Bernd Kolar, Johannes Diwold, and Markus Schöberl
}

\begin{abstract}
We show that the flatness of a nonlinear discretetime system can be checked by computing a unique sequence of involutive distributions. The well-known test for static feedback linearizability is included as a special case. Since the computation of the sequence of distributions requires only the solution of algebraic equations, it allows an efficient implementation in a computer algebra program. In case of a positive result, a flat output can be obtained by straightening out the involutive distributions with the Frobenius theorem.

(C) 2022 IEEE. Personal use of this material is permitted. Permission from IEEE must be obtained for all other uses, in any current or future media, including reprinting/republishing this material for advertising or promotional purposes, creating new collective works, for resale or redistribution to servers or lists, or reuse of any copyrighted component of this work in other works. DOI 10.1109/TAC.2022.3151615
\end{abstract}

Index Terms-Difference flatness, Differential-geometric methods, Discrete-time systems, Feedback linearization, Nonlinear control systems, Normal forms.

\section{INTRODUCTION}

The concept of flatness has been introduced by Fliess, Lévine, Martin and Rouchon in the 1990s for nonlinear continuous-time systems ([1], [2], [3]). For discrete-time systems, flatness can be defined analogously to the continuoustime case in a straightforward way by replacing time derivatives with forward-shifts (see e.g. [4], [5], or [6]). To distinguish both concepts, the terms differential flatness and difference flatness are commonly used. With this definition, like in the continuous-time case, flatness is equivalent to linearizability by an endogenous dynamic feedback as it is defined for discrete-time systems in [7]. We do not consider backward-shifts like in [8] or [9]. According to the terminology of [7], such systems would be linearizable by an exogenous dynamic feedback. The general dynamic feedback linearization problem and related topics like partial feedback linearization are studied e.g. in [10], [11], or [12].

The static feedback linearization problem is a special case of the endogenous dynamic feedback linearization problem and has been solved for discrete-time systems in [13], [14], and [10]. The approach of [13] is similar to the well-known approach of [15] and [16] for continuous-time systems, and allows to check whether a discrete-time system is static feedback linearizable or not by computing a certain sequence of involutive distributions. This test requires only the solution

The first author and the second author have been supported by the Austrian Science Fund (FWF) under grant number P 29964 and P 32151.

All authors are with the Institute of Automatic Control and Control Systems Technology, Johannes Kepler University Linz, Altenbergerstraße 69, 4040 Linz, Austria (e-mail: bernd_kolar@ifac-mail.org, johannes.diwold@jku.at, markus.schoeberl@jku.at). of algebraic equations. Subsequently, a linearizing output can be obtained by straightening out these distributions with the Frobenius theorem, which requires the solution of ODEs.

For checking the flatness of nonlinear discrete-time systems, until now no comparable, computationally similarly efficient test is available. In [17] a sufficient condition for flatness is given, and [5] formulates necessary and sufficient conditions that are analogous to those of [18] for continuoustime systems and rather hard to check. More recently, in [19] we have shown that every flat discrete-time system can be decomposed by coordinate transformations into a subsystem and an endogenous dynamic feedback. The importance of this observation lies in the fact that the complete system is flat if and only if the subsystem is flat. Thus, a repeated application of the decomposition allows to check whether a system with an $n$-dimensional state space is flat or not in at most $n-1$ steps and yields a flat output. However, since the transformations that achieve the decompositions are constructed by straightening out vector fields / distributions with the flow-box theorem / Frobenius theorem, this procedure is still not as computationally efficient as the solution of the static feedback linearization problem in [13]. Therefore, the present paper proposes an a priori test which only checks whether the repeated decompositions are possible or not, without actually performing them. A similar problem is addressed in [20], but with a completely different approach that is based on certain normalized system representations and exterior algebra. Here, in contrast, we introduce a straightforward generalization of the sequence of distributions from the static feedback linearization test of [13]. The difference to the sequence of [13] is that in every step of its construction we do not proceed with the previously constructed distribution itself but rather with its largest "projectable" subdistribution. Like in [13] the proposed sequence of distributions is unique, allows an efficient computation, and the system is flat if and only if the dimension of the last distribution is equal to the dimension of the state space. Thus, we separate the problem of checking flatness from the problem of finding a flat output in the same way as it is possible for static feedback linearizability. If the test yields a positive result, a flat output can be obtained by straightening the distributions out with the Frobenius theorem.

The paper is organized as follows: In Section I and Section III we recapitulate the concept of flatness for discrete-time systems and provide some background on projectable vector fields and distributions, which is the mathematical foundation for our conditions. In particular, we prove that the largest projectable subdistribution of a given distribution is unique and show how it can be computed. In Section IV we present our main result: We introduce a sequence of distributions 
which generalizes the sequence of distributions from the static feedback linearization test, and show that it gives rise to necessary and sufficient conditions for flatness. An example in Section $\mathrm{V}$ illustrates our results.

\section{Discrete-Time Systems And Flatness}

In this contribution we consider discrete-time systems

$$
x^{i,+}=f^{i}(x, u), \quad i=1, \ldots, n
$$

in state representation with $\operatorname{dim}(x)=n, \operatorname{dim}(u)=m$, and smooth functions $f^{i}(x, u)$ that satisfy the submersivity condition

$$
\operatorname{rank}\left(\partial_{(x, u)} f\right)=n .
$$

Submersivity is a usual assumption in the discrete-time literature, and since it is necessary for accessibility (see e.g. [21]), it is actually no restriction 1 Geometrically, the system (1) can be interpreted as a map

$$
f: \mathcal{X} \times \mathcal{U} \rightarrow \mathcal{X}^{+}
$$

from a manifold $\mathcal{X} \times \mathcal{U}$ with coordinates $(x, u)$ to a manifold $\mathcal{X}^{+}$with coordinates $x^{+}$. The condition (2) ensures that this map is a submersion and therefore locally surjective. The notation with a superscript + is used to denote the forwardshift of the corresponding variable. For the inputs and flat outputs we also need higher forward-shifts, and use a subscript in brackets. For instance, $u_{[\alpha]}$ denotes the $\alpha$-th forward-shift of $u$. To keep formulas short and readable we use the Einstein summation convention, and in order to avoid mathematical subtleties we also assume that all functions are smooth. Furthermore, since we use the inverse- and the implicit function theorem, the flow-box theorem, and the Frobenius theorem, it is important to emphasize that all our results are only local. Thus, in order not to loose localness, we have to ensure that the value of $x^{+}$determined by (1) is sufficiently close to $x$. Since the map (1) is continuous, this can be achieved by considering only a sufficiently small neighborhood of an equilibrium point $\left(x_{0}, u_{0}\right)$. This is a common practice in the discrete-time literature, see e.g. [22]. For instance, the closely related discrete-time static feedback linearization problem is also considered around an equilibrium point, see [13] or [23]. For continuous-time systems such a problem does not appear, since by considering a sufficiently small time interval it can always be ensured that the solution stays arbitrarily close to the initial state.

In the following, we summarize the concept of difference flatness. For this purpose, we introduce a space with coordinates $\left(x, u, u_{[1]}, u_{[2]}, \ldots\right)$ and the forward-shift operator $\delta_{x u}$, which acts on a function $g$ according to the rule

$$
\delta_{x u}\left(g\left(x, u, u_{[1]}, u_{[2]}, \ldots\right)\right)=g\left(f(x, u), u_{[1]}, u_{[2]}, u_{[3]}, \ldots\right) \text {. }
$$

A repeated application of $\delta_{x u}$ is denoted by $\delta_{x u}^{\alpha}$. Since in an equilibrium $\left(x_{0}, u_{0}\right)$ the input is kept constant, in this framework it corresponds to a point $\left(x_{0}, u_{0}, u_{0}, u_{0}, \ldots\right)$.

\footnotetext{
${ }^{1}$ In [8], discrete-time flatness is considered also for non-submersive and hence non-accessible systems. However, without accessibility, the practical applicability is significantly reduced.
}

Definition 1. The system (1) is said to be flat around an equilibrium $\left(x_{0}, u_{0}\right)$, if the $n+m$ coordinate functions $x$ and $u$ can be expressed locally by an m-tuple of functions

$$
y^{j}=\varphi^{j}\left(x, u, u_{[1]}, \ldots, u_{[q]}\right), \quad j=1, \ldots, m
$$

and their forward-shifts

$$
\begin{aligned}
y_{[1]} & =\delta_{x u}\left(\varphi\left(x, u, u_{[1]}, \ldots, u_{[q]}\right)\right) \\
y_{[2]} & =\delta_{x u}^{2}\left(\varphi\left(x, u, u_{[1]}, \ldots, u_{[q]}\right)\right) \\
& \vdots
\end{aligned}
$$

up to some finite order. The m-tuple (4) is called a flat output.

With this definition, flatness is equivalent to endogenous dynamic feedback linearizability as it is defined in [7]. The representation of $x$ and $u$ by the flat output and its forwardshifts is unique, and has the form 2

$$
\begin{aligned}
x^{i} & =F_{x}^{i}\left(y_{[0, R-1]}\right), & & i=1, \ldots, n \\
u^{j} & =F_{u}^{j}\left(y_{[0, R]}\right), & & j=1, \ldots, m .
\end{aligned}
$$

Further details can be found e.g. in [19]. For the proof of our main result, the necessary and sufficient condition of Theorem 4 in Section IV we need in particular the following two lemmas.

Lemma 1. ([6], [19]) A system of the form

$$
\begin{aligned}
& x_{1}^{i_{1},+}=f_{1}^{i_{1}}\left(x_{1}, x_{2}, u_{1}\right), \quad i_{1}=1, \ldots, n-m_{2} \\
& x_{2}^{i_{2},+}=f_{2}^{i_{2}}\left(x_{1}, x_{2}, u_{1}, u_{2}\right), \quad i_{2}=1, \ldots, m_{2}
\end{aligned}
$$

with $\operatorname{dim}\left(u_{2}\right)=\operatorname{dim}\left(x_{2}\right)=m_{2}$ and $\operatorname{rank}\left(\partial_{u} f\right)=\operatorname{dim}(u)=$ $m$ is flat if and only if the subsystem

$$
x_{1}^{+}=f_{1}\left(x_{1}, x_{2}, u_{1}\right)
$$

with the $m$ inputs $\left(x_{2}, u_{1}\right)$ is flat.

The equations $x_{2}^{+}=f_{2}\left(x_{1}, x_{2}, u_{1}, u_{2}\right)$ of (6) can be interpreted as an endogenous dynamic feedback for the subsystem (7). This is in accordance with the fact that applying or removing an endogenous dynamic feedback has no effect on the flatness of a system. It is important to note that the Jacobian matrix $\partial_{\left(x_{2}, u_{1}\right)} f_{1}$ does not necessarily have rank $m$, which means that the subsystem (7) may have redundant inputs. However, redundant inputs can be eliminated by suitable transformations: For a system (1) with $\operatorname{rank}\left(\partial_{u} f\right)=\hat{m}<m$, there always exists an input transformation $(\hat{u}, \tilde{u})=\Phi_{u}(x, u)$ with $\operatorname{dim}(\hat{u})=\hat{m}$ that eliminates $m-\hat{m}$ redundant inputs $\tilde{u}$. There is a simple connection between a flat output of the transformed system with $\hat{m}$ inputs, and the original system (1) with $m$ inputs.

Lemma 2. ([19]) Consider a system (11) with $\operatorname{rank}\left(\partial_{u} f\right)=$ $\hat{m}<m$, and an input transformation $(\hat{u}, \tilde{u})=\Phi_{u}(x, u)$ with $\operatorname{dim}(\hat{u})=\hat{m}$ that eliminates $m-\hat{m}$ redundant inputs $\tilde{u}$. If an $\hat{m}$-tuple $\hat{y}$ is a flat output of the transformed system

$$
x^{i,+}=\hat{f}^{i}(x, \hat{u}), \quad i=1, \ldots, n
$$

\footnotetext{
${ }^{2}$ The multi-index $R=\left(r_{1}, \ldots, r_{m}\right)$ contains the number of forward-shifts of each component of the flat output which is needed to express $x$ and $u$, and $y[0, R]$ is an abbreviation for $y$ and its forward-shifts up to order $R$.
} 
with the $\hat{m}$ inputs $\hat{u}$, then the $m$-tuple $y=(\hat{y}, \tilde{u})$ is a flat output of the original system (1) with the $m$ inputs $u$.

Thus, redundant inputs are candidates for components of a flat output.

\section{Projectable Vector Fields and Distributions}

The necessary and sufficient conditions for flatness that we derive in Section IV are based on the concept of projectable vector fields and distributions. In the following, we give a brief overview and refer to [19] or [24] for further details.

We call a vector field

$$
v=v_{x}^{i}(x, u) \partial_{x^{i}}+v_{u}^{j}(x, u) \partial_{u^{j}}
$$

on $\mathcal{X} \times \mathcal{U}$ "projectable" with respect to the map (3), if a pointwise application of the tangent map $f_{*}: \mathcal{T}(\mathcal{X} \times \mathcal{U}) \rightarrow \mathcal{T}\left(\mathcal{X}^{+}\right)$ yields a well-defined vector field

$$
w=w^{i}\left(x^{+}\right) \partial_{x^{i,+}}
$$

on $\mathcal{X}^{+}$. In this case, the components of (8) and (9) meet

$$
w^{i}\left(x^{+}\right) \circ f(x, u)=\partial_{x^{k}} f^{i} v_{x}^{k}(x, u)+\partial_{u^{j}} f^{i} v_{u}^{j}(x, u),
$$

$i=1, \ldots, n$. The vector fields are said to be $f$-related and we write $w=f_{*}(v)$. Checking whether a vector field (8) is projectable or not is a simple task if we introduce coordinates

$$
\begin{aligned}
\theta^{i} & =f^{i}(x, u), \quad i=1, \ldots, n \\
\xi^{j} & =h^{j}(x, u), \quad j=1, \ldots, m
\end{aligned}
$$

on $\mathcal{X} \times \mathcal{U}$ which are adapted to the "fibration" (foliation) determined by the map (3). The $m$ functions $h^{j}(x, u)$ must be chosen such that the Jacobian matrix of the right-hand side of (10) is regular. Because of the submersivity condition (2), this is always possible and can be achieved e.g. by choosing suitable components of $x$ or $u$. With coordinates $(\theta, \xi)$ on $\mathcal{X} \times \mathcal{U}$, the map (3) has the simple form

$$
x^{i,+}=\theta^{i}, \quad i=1, \ldots, n .
$$

All points of $\mathcal{X} \times \mathcal{U}$ with the same value of $\theta$ belong to the same fibre and are mapped to the same point of $\mathcal{X}^{+}$, regardless of the value of the fibre coordinates $\xi$. In adapted coordinates, a vector field (8) on $\mathcal{X} \times \mathcal{U}$ has in general the form

$$
v=a^{i}(\theta, \xi) \partial_{\theta^{i}}+b^{j}(\theta, \xi) \partial_{\xi^{j}},
$$

and because of (11) an application of the tangent map $f_{*}$ yields

$$
f_{*}(v)=a^{i}(\theta, \xi) \partial_{x^{i,+}} .
$$

Obviously, the pointwise pushforward (13) of (12) induces a well-defined vector field on $\mathcal{X}^{+}$if and only if the functions $a^{i}$ are independent of the coordinates $\xi$. In this case, replacing $\theta$ by $x^{+}$yields the vector field (9). In summary, a vector field (8) is projectable if and only if in adapted coordinates (10) it has the form

$$
a^{i}(\theta) \partial_{\theta^{i}}+b^{j}(\theta, \xi) \partial_{\xi^{j}},
$$

and the corresponding vector field (9) is given by

$$
a^{i}\left(x^{+}\right) \partial_{x^{i,+}} .
$$

Similar to projectable vector fields, we call a distribution $D$ on $\mathcal{X} \times \mathcal{U}$ "projectable" if it admits a basis that consists of projectable vector fields (not every vector field contained in a projectable distribution has to be projectable itself). The existence of such a basis ensures that the pushforward $f_{*}(D)$ of a projectable distribution is a well-defined distribution on $\mathcal{X}^{+}$. Moreover, since the Lie brackets $\left[v_{1}, v_{2}\right]$ and $\left[w_{1}, w_{2}\right]$ of two pairs $v_{1}, w_{1}$ and $v_{2}, w_{2}$ of $f$-related vector fields are again $f$-related, i.e. $f_{*}\left[v_{1}, v_{2}\right]=\left[w_{1}, w_{2}\right]$, the pushforward of an involutive projectable distribution is again an involutive distribution. The following theorem is essential for the uniqueness of the sequence of distributions introduced in Section IV.

Theorem 1. The largest projectable subdistribution $D \subset E$ of a distribution $E$ on $\mathcal{X} \times \mathcal{U}$ is uniquely determined. If $E$ is involutive, then $D$ is also involutive.

Proof. Introduce adapted coordinates (10) on $\mathcal{X} \times \mathcal{U}$, and construct a new basis for $E$ which contains as many projectable vector fields (14) as possible. These projectable vector fields are a basis for the largest projectable subdistribution $D$. Every other projectable vector field in $E$ can be written as linear combination of these vector fields. If the distribution $E$ is involutive, then all pairwise Lie brackets of the basis vector fields of the projectable subdistribution $D \subset E$ must be contained in $E$. However, since the basis vector fields of $D$ are projectable, the Lie brackets are again projectable vector fields (see above). Since by construction $D$ contains all projectable vector fields of $E$, the subdistribution $D$ is involutive itself.

In adapted coordinates (10), the computation of the largest projectable subdistribution of a given $d$-dimensional distribution requires only algebraic manipulations. It is convenient to introduce in a first step a "normalized" basis consisting of vector fields

$$
\begin{gathered}
v_{k}=\partial_{\theta^{k}}+a_{k}^{\bar{d}+1}(\theta, \xi) \partial_{\theta^{\bar{d}+1}}+\ldots+a_{k}^{n}(\theta, \xi) \partial_{\theta^{n}} \\
+b_{k}^{1}(\theta, \xi) \partial_{\xi^{1}}+\ldots+b_{k}^{m}(\theta, \xi) \partial_{\xi^{m}},
\end{gathered}
$$

$k=1, \ldots, \bar{d}$ and

$$
v_{l}=b_{l}^{1}(\theta, \xi) \partial_{\xi^{1}}+\ldots+b_{l}^{m}(\theta, \xi) \partial_{\xi^{m}},
$$

$l=\bar{d}+1, \ldots, d$, where $d-\bar{d}$ is the dimension of the subdistribution projecting to zero. Obviously, the $d-\bar{d}$ vector fields (16) are already projectable. As shown in the appendix of [19], a linear combination

$$
c^{1}(\theta, \xi) v_{1}+\ldots+c^{\bar{d}}(\theta, \xi) v_{\bar{d}}
$$

of the $\bar{d}$ remaining vector fields 15 is projectable if and only if the coefficients $c^{1}, \ldots, c^{\bar{d}}$ are independent of $\xi$ and meet

$$
\partial_{\xi^{j}} a_{k}^{i}(\theta, \xi) c^{k}(\theta)=0 \quad \forall i=\bar{d}+1, \ldots, n \text { and } j=1, \ldots, m .
$$

In other words, a linear combination (17) is projectable if and only if the column vector of coefficients

$$
\left[\begin{array}{lll}
c^{1}(\theta) & \cdots & c^{\bar{d}}(\theta)
\end{array}\right]^{T}
$$

lies in the kernel of the $(n-\bar{d}) m \times \bar{d}$-matrix

$$
\left[\begin{array}{lll}
\partial_{\xi^{j}} a_{1}^{i}(\theta, \xi) & \cdots & \partial_{\xi^{j}} a_{\bar{d}}^{i}(\theta, \xi)
\end{array}\right]
$$


with $i=\bar{d}+1, \ldots, n$ and $j=1, \ldots, m$. In contrast to [19], we want to find a maximal set of independent solutions (18). This can be achieved by a successive reduction strategy: If we bring the matrix (19) with row manipulations into the form

$$
\left[\begin{array}{cc}
I & R(\theta, \xi) \\
0 & 0
\end{array}\right]
$$

with an identity matrix $I$ and some remaining matrix $R(\theta, \xi)$, (18) can only lie in its kernel if the product of $R(\theta, \xi)$ with the corresponding elements of 18 is independent of $\xi$. The other elements of (18), i.e., those that are multiplied with the identity matrix, are determined by the fact that the sum of the two products must vanish. Obviously, the product with $R(\theta, \xi)$ is independent of $\xi$ if and only if the corresponding part of (18) lies in the kernel of the matrix

$$
\left[\begin{array}{c}
\partial_{\xi^{1}} R(\theta, \xi) \\
\vdots \\
\partial_{\xi^{m}} R(\theta, \xi)
\end{array}\right] .
$$

Thus, we have reduced the original problem with the matrix (19) to a smaller one with the matrix (20). Continuing the procedure yields a maximal set of independent solutions (18). The corresponding linear combinations 17 form a basis for the largest projectable subdistribution.

\section{NECESSARY AND SUFFICIENT CONDITIONS}

Except for special situations as in [9] with switched systems, verifying whether a given output (4) is a flat output or not is in principle a straightforward task (with a restriction to some upper limit for the number of shifts in (5)). In the following, we address the significantly more difficult problem of checking whether there exists a flat output or not.

In [19] it has been shown that every flat discrete-time system satisfies the following necessary condition.

Theorem 2. The input distribution $\operatorname{span}\left\{\partial_{u}\right\}$ of a flat system (1) with $\operatorname{rank}\left(\partial_{u} f\right)=m$ contains a nontrivial projectable vector field.

In other words, the input distribution contains an at least 1-dimensional projectable subdistribution. Such a projectable vector field or subdistribution can be used to transform the system into the decomposed form (6) of Lemma 11 where the complete system is flat if and only if the subsystem (7) is flat. The required input- and state transformations can be constructed by straightening out the vector field and its pushforward (or the corresponding distributions) by the flowbox theorem / Frobenius theorem. As shown in [19], a repeated application of this decomposition allows to check whether a system (1) is flat or not in at most $n-1$ steps. However, straightening out vector fields / distributions with the flowbox theorem / Frobenius theorem requires the solution of ODEs. For this reason, in the following we introduce a computationally more efficient a priori test, which allows to check whether the repeated decompositions are possible or not without actually performing them. This test relies on sequences of nested distributions on $\mathcal{X} \times \mathcal{U}$ and $\mathcal{X}^{+}$. The construction of these sequences of distributions is based on the map (3) defined by the system equations (1), and the map

$$
\pi: \mathcal{X} \times \mathcal{U} \rightarrow \mathcal{X}^{+}
$$

defined by

$$
x^{i,+}=x^{i}, \quad i=1, \ldots, n .
$$

We assume that the distributions have locally constant dimension.

\section{Algorithm 1.}

Step 0: Define the involutive distributions $\Delta_{0}=0$ on $\mathcal{X}^{+}$ and $E_{0}=\pi_{*}^{-1}\left(\Delta_{0}\right)=\operatorname{span}\left\{\partial_{u}\right\}$ on $\mathcal{X} \times \mathcal{U}$. Then compute the largest subdistribution $D_{0} \subset E_{0}$ which is projectable with respect to the map (3). Because of Theorem $1, D_{0}$ is unique and involutive. Thus, the pushforward $\Delta_{1}=f_{*}\left(D_{0}\right)$ is a welldefined involutive distribution on $\mathcal{X}^{+}$.

Step $k \geq 1$ : Define the involutive distribution

$$
E_{k}=\pi_{*}^{-1}\left(\Delta_{k}\right)
$$

on $\mathcal{X} \times \mathcal{U}$. Because of $\Delta_{k-1} \subset \Delta_{k}$, it satisfies $E_{k-1} \subset E_{k}$. Then compute the largest subdistribution

$$
D_{k} \subset E_{k}
$$

which is projectable with respect to the map (3). Because of Theorem 1] $D_{k}$ is unique and involutive. Moreover, because of $D_{k-1} \subset E_{k-1}$ and $E_{k-1} \subset E_{k}$, it satisfies $D_{k-1} \subset D_{k}$. Thus, the pushforward

$$
\Delta_{k+1}=f_{*}\left(D_{k}\right)
$$

is a well-defined involutive distribution on $\mathcal{X}^{+}$with

$$
\Delta_{k} \subset \Delta_{k+1}
$$

Stop if $\operatorname{dim}\left(\Delta_{\bar{k}+1}\right)=\operatorname{dim}\left(\Delta_{\bar{k}}\right)$ for some $k=\bar{k}$.

Remark 1. The distributions $\Delta_{k}$ are involutive since the pushforward of a projectable and involutive distribution $D_{k-1}$ is again an involutive distribution. Subsequently, the involutivity of $\Delta_{k}$ implies the involutivity of $E_{k}$. For $k=0$ with $E_{0}=\operatorname{span}\left\{\partial_{u}\right\}$, this is obvious. For $k \geq 1$ we know that $\Delta_{k}$ is involutive, and can perform a state transformation $\left(\tilde{x}_{1}, \tilde{x}_{2}\right)=\Phi_{x}(x)$ with $\operatorname{dim}\left(\tilde{x}_{1}\right)=\operatorname{dim}\left(\Delta_{k}\right)$ such that $\Delta_{k}=\operatorname{span}\left\{\partial_{\tilde{x}_{1}^{+}}\right\}$. In these coordinates, $E_{k}=\operatorname{span}\left\{\partial_{\tilde{x}_{1}}, \partial_{u}\right\}$ is straightened out and hence clearly involutive.

Because of (22) and $\operatorname{dim}\left(\mathcal{X}^{+}\right)=n$, the procedure terminates after at most $n$ steps. It yields a unique nested sequence of projectable and involutive distributions

$$
D_{0} \subset D_{1} \subset \ldots \subset D_{\bar{k}-1}
$$

on $\mathcal{X} \times \mathcal{U}$, and a unique nested sequence of involutive distributions

$$
\Delta_{1} \subset \Delta_{2} \subset \ldots \subset \Delta_{\bar{k}}
$$

${ }^{3}$ As in [13] and [23], $\pi_{*}^{-1}(\Delta)$ denotes the inverse image of a distribution $\Delta$ under the tangent map $\pi_{*}$. If $\Delta=\operatorname{span}\left\{v_{1}^{i}\left(x^{+}\right) \partial_{x^{i,+}}, \ldots, v_{d}^{i}\left(x^{+}\right) \partial_{x^{i,+}}\right\}$ is a $d$-dimensional distribution on $\mathcal{X}^{+}$, then $\pi_{*}^{-1}(\Delta)$ is the $(d+m)$ dimensional distribution $\operatorname{span}\left\{v_{1}^{i}(x) \partial_{x^{i}}, \ldots, v_{d}^{i}(x) \partial_{x^{i}}, \partial_{u^{1}}, \ldots, \partial_{u^{m}}\right\}$ on $\mathcal{X} \times \mathcal{U}$ 
on $\mathcal{X}^{+}$, which are related by the condition 21 . Since we have assumed that locally all these distributions have constant dimension, we can define

$$
\rho_{k}=\operatorname{dim}\left(\Delta_{k}\right)-\operatorname{dim}\left(\Delta_{k-1}\right), \quad k \geq 1
$$

with $\operatorname{dim}\left(\Delta_{0}\right)=0$. Since the pushforward of linearly independent, projectable vector fields on $\mathcal{X} \times \mathcal{U}$ does not necessarily yield linearly independent vector fields on $\mathcal{X}^{+}$, it is also important to note that in general

$$
\operatorname{dim}\left(\Delta_{k}\right) \leq \operatorname{dim}\left(D_{k-1}\right)
$$

Therefore, we additionally define

$$
\mu_{k}=\operatorname{dim}\left(D_{k}\right)-\operatorname{dim}\left(\Delta_{k+1}\right)-\underbrace{\left(\operatorname{dim}\left(D_{k-1}\right)-\operatorname{dim}\left(\Delta_{k}\right)\right)}_{\mu_{0}+\ldots+\mu_{k-1}},
$$

$k \geq 1$, which is just the number of linearly independent vector fields $v \in D_{k}$ with $f_{*}(v)=0$ that are not contained in $D_{k-1}$. For $k=0$, in the case $\operatorname{rank}\left(\partial_{u} f\right)=m$ we always have

$$
\mu_{0}=\operatorname{dim}\left(D_{0}\right)-\operatorname{dim}\left(\Delta_{1}\right)=0 .
$$

The sequence (24) generalizes a sequence which was introduced in [13] to check whether a discrete-time system (1) is static feedback linearizable or not.

Theorem 3. A system (1) with $\operatorname{rank}\left(\partial_{u} f\right)=m$ is static feedback linearizable if and only if $D_{k}=E_{k}, k \geq 0$ and $\operatorname{dim}\left(\Delta_{\bar{k}}\right)=n$

Hence, for static feedback linearizability, in every step of Algorithm 1 the complete distribution $E_{k}$ must be projectable. For a proof see [13] or [23]. If we drop the condition $D_{k}=$ $E_{k}$, we get necessary and sufficient conditions for flatness.

Theorem 4. A system (1) with $\operatorname{rank}\left(\partial_{u} f\right)=m$ is flat if and only if $\operatorname{dim}\left(\Delta_{\bar{k}}\right)=n$.

Before we prove Theorem 4, we establish some further properties of the sequences (23) and (24). The basic idea of the proof, however, is to use the distributions (23) and (24) for a stepwise decomposition of the system (1) into subsystems and endogenous dynamic feedbacks exactly like in [19]. In the case $\operatorname{dim}\left(\Delta_{\bar{k}}\right)=n$, the system can be decomposed until only the trivial system is left, which proves that the system is flat. In the case $\operatorname{dim}\left(\Delta_{\bar{k}}\right)<n$, in contrast, there occurs a subsystem which allows no further decomposition. This is, however, a contradiction to the necessary condition for flatness derived in [19].

First, it is important to note that the nested sequence of involutive distributions (24) on $\mathcal{X}^{+}$can be straightened out by a state transformation

$$
\left(\bar{x}_{1}, \ldots, \bar{x}_{\bar{k}}, \bar{x}_{r e s t}\right)=\Phi_{x}(x)
$$

with $\operatorname{dim}\left(\bar{x}_{k}\right)=\rho_{k}, k=1, \ldots, \bar{k}$ such that

$$
\begin{aligned}
\Delta_{1} & =\operatorname{span}\left\{\partial_{\bar{x}_{1}^{+}}\right\} \\
\Delta_{2} & =\operatorname{span}\left\{\partial_{\bar{x}_{1}^{+}}, \partial_{\bar{x}_{2}^{+}}\right\} \\
& \vdots \\
\Delta_{\bar{k}} & =\operatorname{span}\left\{\partial_{\bar{x}_{1}^{+}}, \partial_{\bar{x}_{2}^{+}}, \ldots, \partial_{\bar{x}_{\bar{k}}^{+}}\right\} .
\end{aligned}
$$

In accordance with the transformation law for discrete-time systems, the state transformation (26) is performed both for the variables $x$ and the shifted variables $x^{+}$. Because of (21), the transformed system

$$
\begin{aligned}
\bar{x}_{r e s t}^{+} & =f_{\text {rest }}(\bar{x}, u) \\
\bar{x}_{\bar{k}}^{+} & =f_{\bar{k}}(\bar{x}, u) \\
& \vdots \\
\bar{x}_{2}^{+} & =f_{2}(\bar{x}, u) \\
\bar{x}_{1}^{+} & =f_{1}(\bar{x}, u)
\end{aligned}
$$

meets

$$
\begin{aligned}
f_{*}\left(D_{0}\right) & =\operatorname{span}\left\{\partial_{\bar{x}_{1}^{+}}\right\} \\
f_{*}\left(D_{1}\right) & =\operatorname{span}\left\{\partial_{\bar{x}_{1}^{+}}, \partial_{\bar{x}_{2}^{+}}\right\} \\
& \vdots \\
f_{*}\left(D_{\bar{k}-1}\right) & =\operatorname{span}\left\{\partial_{\bar{x}_{1}^{+}}, \partial_{\bar{x}_{2}^{+}}, \ldots, \partial_{\bar{x}_{\bar{k}}^{+}}\right\} .
\end{aligned}
$$

In these coordinates, the involutive distributions

$$
E_{k}=\pi_{*}^{-1}\left(\Delta_{k}\right)=\operatorname{span}\left\{\partial_{\bar{x}_{k}}, \ldots, \partial_{\bar{x}_{1}}, \partial_{u}\right\},
$$

$k=0, \ldots, \bar{k}-1$ are exactly the input distributions of the subsystems

$$
\begin{aligned}
\bar{x}_{\text {rest }}^{+} & =f_{\text {rest }}\left(\bar{x}_{\text {rest }}, \bar{x}_{\bar{k}}, \ldots, \bar{x}_{1}, u\right) \\
\bar{x}_{\bar{k}}^{+} & =f_{\bar{k}}\left(\bar{x}_{\text {rest }}, \bar{x}_{\bar{k}}, \ldots, \bar{x}_{1}, u\right) \\
& \vdots \\
\bar{x}_{k+1}^{+} & =f_{k+1}\left(\bar{x}_{\text {rest }}, \bar{x}_{\bar{k}}, \ldots, \bar{x}_{1}, u\right)
\end{aligned}
$$

of (27) without the equations for $\left(\bar{x}_{k}, \ldots, \bar{x}_{1}\right)$. Among the inputs $\left(\bar{x}_{k}, \ldots, \bar{x}_{1}, u\right)$ of these subsystems there are of course redundant inputs.

Lemma 3. The rank of the Jacobian matrix

$$
\left[\begin{array}{cccc}
\partial_{\bar{x}_{k}} f_{\text {rest }} & \cdots & \partial_{\bar{x}_{1}} f_{\text {rest }} & \partial_{u} f_{\text {rest }} \\
\partial_{\bar{x}_{k}} f_{\bar{k}} & \cdots & \partial_{\bar{x}_{1}} f_{\bar{k}} & \partial_{u} f_{\bar{k}} \\
\vdots & & \vdots & \vdots \\
\partial_{\bar{x}_{k}} f_{k+1} & \cdots & \partial_{\bar{x}_{1}} f_{k+1} & \partial_{u} f_{k+1}
\end{array}\right]
$$

of the subsystem (29) with respect to its inputs $\left(\bar{x}_{k}, \ldots, \bar{x}_{1}, u\right)$ is given by

$$
m-\left(\operatorname{dim}\left(D_{k}\right)-\operatorname{dim}\left(\Delta_{k+1}\right)\right)=m-\left(\mu_{0}+\ldots+\mu_{k}\right) .
$$

Proof. The Jacobian matrix (30) has

$$
\rho_{k}+\ldots+\rho_{1}+m=\operatorname{dim}\left(\Delta_{k}\right)+m
$$

columns. To prove the lemma, we simply calculate the dimension of its kernel. For the distribution $D_{k} \subset E_{k}$ with $f_{*}\left(D_{k}\right)=\Delta_{k+1}$, there exists a basis that contains $\operatorname{dim}\left(D_{k}\right)-$ $\rho_{k+1}$ vector fields with a pushforward that lies in $\Delta_{k} \subset \Delta_{k+1}$. Written as column vectors, these $\operatorname{dim}\left(D_{k}\right)-\rho_{k+1}$ vector fields lie in the kernel of the Jacobian matrix (30). Thus, the matrix has a kernel of dimension at least $\operatorname{dim}\left(D_{k}\right)-\rho_{k+1}$. However, every vector field $v \in E_{k}$ which lies in the kernel of (30) has a pushforward that lies in $\Delta_{k}$, and because of $f_{*}\left(D_{k-1}\right)=\Delta_{k}$ it is certainly contained in $D_{k}$. Thus, the dimension of the kernel is exactly $\operatorname{dim}\left(D_{k}\right)-\rho_{k+1}$. Subtracting the dimension of the kernel from the number of columns gives the rank

$$
\operatorname{dim}\left(\Delta_{k}\right)+m-\left(\operatorname{dim}\left(D_{k}\right)-\rho_{k+1}\right) .
$$


With $\rho_{k+1}=\operatorname{dim}\left(\Delta_{k+1}\right)-\operatorname{dim}\left(\Delta_{k}\right)$ and the definition 25) of the integers $\mu_{k}$, the result (31) follows.

If the system (1) is static feedback linearizable, then the state transformation (26) transforms the system into a triangular form

$$
\begin{aligned}
\bar{x}_{\bar{k}}^{+} & =f_{\bar{k}}\left(\bar{x}_{\bar{k}}, \bar{x}_{\bar{k}-1}\right) \\
& \vdots \\
\bar{x}_{2}^{+} & =f_{2}\left(\bar{x}_{\bar{k}}, \bar{x}_{\bar{k}-1}, \ldots, \bar{x}_{1}\right) \\
\bar{x}_{1}^{+} & =f_{1}\left(\bar{x}_{\bar{k}}, \bar{x}_{\bar{k}-1}, \ldots, \bar{x}_{1}, u\right),
\end{aligned}
$$

see also [13] and [23]. The reason is that with $D_{k}=E_{k}, k \geq 0$ straightening out the sequence (24) simultaneously straightens out the sequence $E_{0} \subset E_{1} \subset \ldots \subset E_{\bar{k}-1}$. Consequently, $D_{k}=\operatorname{span}\left\{\partial_{\bar{x}_{k}}, \ldots, \partial_{\bar{x}_{1}}, \partial_{u}\right\}, k=0, \ldots, \bar{k}-1$. Evaluating the condition (28) shows that the transformed system (27) with $\operatorname{dim}\left(\bar{x}_{\text {rest }}\right)=0$ must have the triangular form (32). However, if $D_{k}=E_{k}$ does not hold for all $k=0, \ldots, \bar{k}-1$, then the state transformation (26) that straightens out the sequence (24) does in general not straighten out the sequence (23).

\section{A. Proof of Theorem 4}

In the following, we prove Theorem 4 for the system 27) after the state transformation (26), i.e., we assume that the distributions (24) have already been straightened out. The idea of the proof is to straighten out the sequence (23) in $\bar{k}$ steps with coordinate transformations on $\mathcal{X} \times \mathcal{U}$ that can be interpreted as input transformations for the subsystems (29), $k=0, \ldots, \bar{k}-1$. These transformations result in a sequence of decomposed subsystems, and show inductively that the complete system (27) is flat if and only if the subsystem with $f_{\text {rest }}$ is flat.

First, we decompose the system (27) by straightening out $D_{0}$. Because of $D_{0} \subset E_{0}=\operatorname{span}\left\{\partial_{u}\right\}$, there exists an input transformation

$$
\left(\eta_{0}, \hat{z}_{0}\right)=\Phi_{0}(\bar{x}, u)
$$

with inverse $u=\hat{\Phi}_{0}\left(\bar{x}, \eta_{0}, \hat{z}_{0}\right)$ such that

$$
D_{0}=\operatorname{span}\left\{\partial_{\hat{z}_{0}}\right\} .
$$

Because of $f_{*}\left(D_{0}\right)=\operatorname{span}\left\{\partial_{\bar{x}_{1}^{+}}\right\}$, in these coordinates the functions $f_{2}, \ldots, f_{\bar{k}}, f_{\text {rest }}$ are independent of $\hat{z}_{0}$, i.e.,

$$
\begin{aligned}
\bar{x}_{\text {rest }}^{+} & =f_{\text {rest }}\left(\bar{x}_{\text {rest }}, \bar{x}_{\bar{k}}, \ldots, \bar{x}_{2}, \bar{x}_{1}, \eta_{0}\right) \\
\bar{x}_{\bar{k}}^{+} & =f_{\bar{k}}\left(\bar{x}_{\text {rest }}, \bar{x}_{\bar{k}}, \ldots, \bar{x}_{2}, \bar{x}_{1}, \eta_{0}\right) \\
& \vdots \\
\bar{x}_{2}^{+} & =f_{2}\left(\bar{x}_{\text {rest }}, \bar{x}_{\bar{k}}, \ldots, \bar{x}_{2}, \bar{x}_{1}, \eta_{0}\right) \\
\bar{x}_{1}^{+} & =f_{1}\left(\bar{x}_{\text {rest }}, \bar{x}_{\bar{k}}, \ldots, \bar{x}_{2}, \bar{x}_{1}, \eta_{0}, \hat{z}_{0}\right) .
\end{aligned}
$$

Since the system (27) does not have redundant inputs,

$$
\operatorname{rank}\left(\partial_{\hat{z}_{0}} f_{1}\right)=\operatorname{dim}\left(\hat{z}_{0}\right)=\rho_{1}
$$

holds. Thus, the system is flat if and only if the subsystem $f_{2}, \ldots, f_{\bar{k}}, f_{\text {rest }}$ with the inputs $\left(\bar{x}_{1}, \eta_{0}\right)$ is flat (cf. Lemma 11. Next, because of the rank condition of Lemma 3, we can eliminate $\mu_{1}$ redundant inputs $y_{1}$ of this subsystem by an input transformation

$$
\left(\zeta_{1}, y_{1}\right)=\Psi_{1}\left(\bar{x}_{r e s t}, \bar{x}_{\bar{k}}, \ldots, \bar{x}_{2}, \bar{x}_{1}, \eta_{0}\right)
$$

with inverse $\left(\bar{x}_{1}, \eta_{0}\right)=\hat{\Psi}_{1}\left(\bar{x}_{\text {rest }}, \bar{x}_{\bar{k}}, \ldots, \bar{x}_{2}, \zeta_{1}, y_{1}\right)$. This yields

$$
\begin{aligned}
\bar{x}_{r e s t}^{+} & =f_{\text {rest }}\left(\bar{x}_{\text {rest }}, \bar{x}_{\bar{k}}, \ldots, \bar{x}_{2}, \zeta_{1}\right) \\
\bar{x}_{\bar{k}}^{+} & =f_{\bar{k}}\left(\bar{x}_{\text {rest }}, \bar{x}_{\bar{k}}, \ldots, \bar{x}_{2}, \zeta_{1}\right) \\
& \vdots \\
\bar{x}_{2}^{+} & =f_{2}\left(\bar{x}_{\text {rest }}, \bar{x}_{\bar{k}}, \ldots, \bar{x}_{2}, \zeta_{1}\right) \\
\bar{x}_{1}^{+} & =f_{1}\left(\bar{x}_{\text {rest }}, \bar{x}_{\bar{k}}, \ldots, \bar{x}_{2}, \zeta_{1}, y_{1}, \hat{z}_{0}\right)
\end{aligned}
$$

with $\operatorname{dim}\left(\zeta_{1}\right)=m-\left(\operatorname{dim}\left(D_{1}\right)-\operatorname{dim}\left(\Delta_{2}\right)\right)$ according to (31). Now that all redundant inputs are eliminated, we decompose the subsystem $f_{2}, \ldots, f_{\bar{k}}, f_{\text {rest }}$ by straightening out

$$
D_{1} \subset E_{1}=\operatorname{span}\left\{\partial_{\hat{z}_{0}}, \partial_{y_{1}}, \partial_{\zeta_{1}}\right\} .
$$

Because of $D_{0} \subset D_{1}$ and $f_{*}\left(\partial_{y_{1}}\right) \subset \operatorname{span}\left\{\partial_{\bar{x}_{1}^{+}}\right\}$we have

$$
\operatorname{span}\left\{\partial_{\hat{z}_{0}}, \partial_{y_{1}}\right\} \subset D_{1},
$$

and consequently $D_{1}$ has a basis of the form

$$
\begin{array}{ll}
\partial_{\hat{z}_{0}^{i_{0}}}, & i_{0}=1, \ldots, \rho_{1} \\
\partial_{y_{1}^{j_{1}}}, & j_{1}=1, \ldots, \mu_{1}
\end{array}
$$

$$
\sum_{l=1}^{\operatorname{dim}\left(\zeta_{1}\right)} v_{i_{1}}^{l}\left(\bar{x}_{\text {rest }}, \ldots, \bar{x}_{2}, \zeta_{1}, y_{1}, \hat{z}_{0}\right) \partial_{\zeta_{1}^{l}}, \quad i_{1}=1, \ldots, \rho_{2} .
$$

Up to a renumbering of the components of $\zeta_{1}$, there even exists a basis

$$
\begin{aligned}
& \partial_{\hat{z}_{0}^{i_{0}}}, i_{0}=1, \ldots, \rho_{1} \\
& \partial_{y_{1}^{j_{1}}}, j_{1}=1, \ldots, \mu_{1} \\
& \partial_{\zeta_{1}^{i_{1}}}+\sum_{l=\rho_{2}+1}^{\operatorname{dim}\left(\zeta_{1}\right)} \hat{v}_{i_{1}}^{l}\left(\bar{x}_{\text {rest }}, \ldots, \bar{x}_{2}, \zeta_{1}\right) \partial_{\zeta_{1}^{l}}, \quad i_{1}=1, \ldots, \rho_{2},
\end{aligned}
$$

which, written in matrix form, contains a block with an identity matrix. Thus, the involutivity of $D_{1}$ implies that all pairwise Lie brackets of the vector fields vanish. This in turn implies that the coefficients of the last $\rho_{2}$ vector fields are independent of $\hat{z}_{0}$ and $y_{1}$. Therefore, these vector fields can be straightened out by a transformation of the form

$$
\left(\eta_{1}, \hat{z}_{1}\right)=\Phi_{1}\left(\bar{x}_{r e s t}, \bar{x}_{\bar{k}}, \ldots, \bar{x}_{2}, \zeta_{1}\right)
$$

with inverse $\zeta_{1}=\hat{\Phi}_{1}\left(\bar{x}_{\text {rest }}, \bar{x}_{\bar{k}}, \ldots, \bar{x}_{2}, \eta_{1}, \hat{z}_{1}\right)$, which can be interpreted as an input transformation for the subsystem $f_{2}, \ldots, f_{\bar{k}}, f_{\text {rest }}$. In new coordinates we have

$$
D_{1}=\operatorname{span}\left\{\partial_{\hat{z}_{0}}, \partial_{y_{1}}, \partial_{\hat{z}_{1}}\right\} \text {, }
$$

and because of $f_{*}\left(D_{1}\right)=\operatorname{span}\left\{\partial_{\bar{x}_{1}^{+}}, \partial_{\bar{x}_{2}^{+}}\right\}$the functions $f_{3}, \ldots, f_{\bar{k}}, f_{\text {rest }}$ are independent of $\hat{z}_{0}, y_{1}$, and $\hat{z}_{1}$. Thus,

$$
\begin{aligned}
\bar{x}_{\text {rest }}^{+} & =f_{\text {rest }}\left(\bar{x}_{\text {rest }}, \bar{x}_{\bar{k}}, \ldots, \bar{x}_{2}, \eta_{1}\right) \\
\bar{x}_{\bar{k}}^{+} & =f_{\bar{k}}\left(\bar{x}_{\text {rest }}, \bar{x}_{\bar{k}}, \ldots, \bar{x}_{2}, \eta_{1}\right) \\
& \vdots \\
\bar{x}_{3}^{+} & =f_{3}\left(\bar{x}_{\text {rest }}, \bar{x}_{\bar{k}}, \ldots, \bar{x}_{2}, \eta_{1}\right) \\
\bar{x}_{2}^{+} & =f_{2}\left(\bar{x}_{\text {rest }}, \bar{x}_{\bar{k}}, \ldots, \bar{x}_{2}, \eta_{1}, \hat{z}_{1}\right) \\
\bar{x}_{1}^{+} & =f_{1}\left(\bar{x}_{\text {rest }}, \bar{x}_{\bar{k}}, \ldots, \bar{x}_{2}, \eta_{1}, \hat{z}_{1}, y_{1}, \hat{z}_{0}\right)
\end{aligned}
$$

and

$$
\operatorname{rank}\left(\partial_{\hat{z}_{1}} f_{2}\right)=\operatorname{dim}\left(\hat{z}_{1}\right)=\rho_{2} .
$$


Consequently, the system is flat if and only if the subsystem $f_{3}, \ldots, f_{\bar{k}}, f_{\text {rest }}$ with the inputs $\left(\bar{x}_{2}, \eta_{1}\right)$ is flat. In the following steps, we proceed analogously. First, we eliminate all redundant inputs of the subsystem $f_{k+1}, \ldots, f_{\bar{k}}, f_{\text {rest }}, k \geq 2$. Subsequently, we decompose the subsystem by straightening out the distribution $D_{k}$ with a transformation that can be interpreted as an input transformation for the subsystem. Continuing this procedure until $k=\bar{k}-1$ introduces new coordinates on $\mathcal{X} \times \mathcal{U}$ such that the map (27) has the form

$$
\begin{aligned}
\bar{x}_{\text {rest }}^{+} & =f_{\text {rest }}\left(\bar{x}_{\text {rest }}, \bar{x}_{\bar{k}}, \eta_{\bar{k}-1}\right) \\
\bar{x}_{\bar{k}}^{+} & =f_{\bar{k}}\left(\bar{x}_{\text {rest }}, \bar{x}_{\bar{k}}, \eta_{\bar{k}-1}, \hat{z}_{\bar{k}-1}\right) \\
& \vdots \\
\bar{x}_{2}^{+} & =f_{2}\left(\bar{x}_{\text {rest }}, \bar{x}_{\bar{k}}, \eta_{\bar{k}-1}, \hat{z}_{\bar{k}-1}, y_{\bar{k}-1}, \ldots, \hat{z}_{2}, y_{2}, \hat{z}_{1}\right) \\
\bar{x}_{1}^{+} & =f_{1}\left(\bar{x}_{\text {rest }}, \bar{x}_{\bar{k}}, \eta_{\bar{k}-1}, \hat{z}_{\bar{k}-1}, y_{\bar{k}-1}, \ldots, \hat{z}_{1}, y_{1}, \hat{z}_{0}\right),
\end{aligned}
$$

and shows by a repeated application of Lemma 1 that the complete system is flat if and only if the subsystem

$$
\bar{x}_{\text {rest }}^{+}=f_{\text {rest }}\left(\bar{x}_{\text {rest }}, \bar{x}_{\bar{k}}, \eta_{\bar{k}-1}\right)
$$

with the inputs $\left(\bar{x}_{\bar{k}}, \eta_{\bar{k}-1}\right)$ is flat.

In the case $\operatorname{dim}\left(\Delta_{\bar{k}}\right)=n$, because of $\operatorname{dim}\left(\bar{x}_{\text {rest }}\right)=0$ the subsystem (33) is an empty system with inputs $\left(\bar{x}_{\bar{k}}, \eta_{\bar{k}-1}\right)$. Therefore, the complete system is flat, and

$$
y=\left(y_{\bar{k}}, y_{\bar{k}-1}, \ldots, y_{1}\right)
$$

with $y_{\bar{k}}=\left(\bar{x}_{\bar{k}}, \eta_{\bar{k}-1}\right)$ is a flat output. The flat output (34) consists of the inputs of the (empty) system (33), and the redundant inputs $\left(y_{\bar{k}-1}, \ldots, y_{1}\right)$ of the subsystems that have been eliminated during the repeated decompositions (cf. Lemma 2). The flat output in original coordinates can be obtained by applying the inverse coordinate transformations.

For the case $\operatorname{dim}\left(\Delta_{\bar{k}}\right)<n$, we show by contradiction that the subsystem (33) with $\operatorname{dim}\left(\bar{x}_{\text {rest }}\right)>0$ cannot be flat. First, we eliminate all redundant inputs by an input transformation

$$
\left(\zeta_{\bar{k}}, y_{\bar{k}}\right)=\Psi_{\bar{k}}\left(\bar{x}_{r e s t}, \bar{x}_{\bar{k}}, \eta_{\bar{k}-1}\right)
$$

with inverse $\left(\bar{x}_{\bar{k}}, \eta_{\bar{k}-1}\right)=\hat{\Psi}_{\bar{k}}\left(\bar{x}_{r e s t}, \zeta_{\bar{k}}, y_{\bar{k}}\right)$. If the resulting system

$$
\bar{x}_{\text {rest }}^{+}=f_{\text {rest }}\left(\bar{x}_{\text {rest }}, \zeta_{\bar{k}}\right)
$$

would be flat, then according to the necessary condition of Theorem 2 there would exist a nontrivial vector field $v^{l}\left(\bar{x}_{\text {rest }}, \zeta_{\bar{k}}\right) \partial_{\zeta_{\bar{k}}^{l}}$ which is projectable with respect to the subsystem (35). With respect to the complete system, such a vector field would be contained in the largest projectable subdistribution $D_{\bar{k}} \subset E_{\bar{k}}$, and accordingly the dimension of $\Delta_{\bar{k}+1}=f_{*}\left(D_{\bar{k}}\right)$ would be larger than the dimension of $\Delta_{\bar{k}}=$ $f_{*}\left(D_{\bar{k}-1}\right)$. However, because of $\operatorname{dim}\left(\Delta_{\bar{k}+1}\right)=\operatorname{dim}\left(\Delta_{\bar{k}}\right)$ such a vector field does not exist.

Remark 2. The Frobenius theorem, which is used for straightening out the distributions (23) and (24), guarantees only local results. Thus, it should be noted that if the distribution $D_{0}$ is considered locally around some point $(\bar{x}, \bar{u}) \in \mathcal{X} \times \mathcal{U}$, then the distributions $\Delta_{1}=f_{*}\left(D_{0}\right)$ and $D_{1} \subset E_{1}=\pi_{*}^{-1}\left(\Delta_{1}\right)$ are defined locally around the points $f(\bar{x}, \bar{u}) \in \mathcal{X}^{+}$and $(f(\bar{x}, \bar{u}), \bar{u}) \in \mathcal{X} \times \mathcal{U}$, respectively. Likewise, $\Delta_{2}$ and $D_{2}$ are defined around $f(f(\bar{x}, \bar{u}), \bar{u}) \in \mathcal{X}^{+}$and $(f(f(\bar{x}, \bar{u}), \bar{u}), \bar{u}) \in$
$\mathcal{X} \times \mathcal{U}$ (and so on). However, considering only a sufficiently small neighborhood of an equilibrium $\left(x_{0}, u_{0}\right)$ ensures by $x_{0}=f\left(x_{0}, u_{0}\right)$ and the continuity of $f$ that the points $\bar{x}, f(\bar{x}, \bar{u}), f(f(\bar{x}, \bar{u}), \bar{u}), \ldots$ are sufficiently close, such that the regions of validity of all coordinate transformations overlap. In practice, the regions of validity of the coordinate transformations - and consequently the region of validity of the proof of Theorem 4-can of course be quite large.

\section{EXAMPLE}

In [19], we have already shown that the system

$$
\begin{aligned}
& x^{1,+}=\frac{x^{2}+x^{3}+3 x^{4}}{u^{1}+2 u^{2}+1} \\
& x^{2,+}=x^{1}\left(x^{3}+1\right)\left(u^{1}+2 u^{2}-3\right)+x^{4}-3 u^{2} \\
& x^{3,+}=u^{1}+2 u^{2} \\
& x^{4,+}=x^{1}\left(x^{3}+1\right)+u^{2}
\end{aligned}
$$

is flat around the equilibrium $\left(x_{0}, u_{0}\right)=(0,0)$ by repeated transformations into subsystems and endogenous dynamic feedbacks. In the following, we prove its flatness again by simply computing the sequence of distributions (24) and applying Theorem 4 i.e., without actually performing any decompositions like in [19]. In the first step of Algorithm 1 , we have to calculate the largest projectable subdistribution of the distribution $E_{0}=\operatorname{span}\left\{\partial_{u^{1}}, \partial_{u^{2}}\right\}$. For this purpose, we introduce adapted coordinates (10) on $\mathcal{X} \times \mathcal{U}$. After the transformation

$$
\begin{aligned}
& \theta^{1}=f^{1}(x, u) \\
& \theta^{2}=f^{2}(x, u) \\
& \theta^{3}=f^{3}(x, u) \\
& \theta^{4}=f^{4}(x, u)
\end{aligned} \quad \quad \xi^{1}=x^{1}=x^{3},
$$

the vector fields $\partial_{u^{1}}$ and $\partial_{u^{2}}$ are given by

$$
-\frac{\theta^{1}}{\theta^{3}+1} \partial_{\theta^{1}}+\xi^{1}\left(\xi^{2}+1\right) \partial_{\theta^{2}}+\partial_{\theta^{3}}
$$

and

$$
-2 \frac{\theta^{1}}{\theta^{3}+1} \partial_{\theta^{1}}+\left(2 \xi^{1}\left(\xi^{2}+1\right)-3\right) \partial_{\theta^{2}}+2 \partial_{\theta^{3}}+\partial_{\theta^{4}} .
$$

Because of the presence of the fibre coordinates $\xi^{1}$ and $\xi^{2}$, neither $\partial_{u^{1}}$ nor $\partial_{u^{2}}$ itself is projectable. However, the linear combination $-2 \partial_{u^{1}}+\partial_{u^{2}}$ reads in adapted coordinates as $-3 \partial_{\theta^{2}}+\partial_{\theta^{4}}$, and is therefore a projectable vector field. Thus, the largest projectable subdistribution is given by

$$
D_{0}=\operatorname{span}\left\{-2 \partial_{u^{1}}+\partial_{u^{2}}\right\} .
$$

The pushforward $f_{*}\left(D_{0}\right)$ is the involutive distribution

$$
\Delta_{1}=\operatorname{span}\left\{-3 \partial_{x^{2,+}}+\partial_{x^{4,+}}\right\}
$$

on $\mathcal{X}^{+}$with $\operatorname{dim}\left(\Delta_{1}\right)=\operatorname{dim}\left(D_{0}\right)=1$. In the second step, we have to determine the largest projectable subdistribution of

$$
E_{1}=\operatorname{span}\left\{-3 \partial_{x^{2}}+\partial_{x^{4}}, \partial_{u^{1}}, \partial_{u^{2}}\right\} .
$$

In adapted coordinates (37), it can be verified that the complete distribution is projectable, i.e., $D_{1}=E_{1}$. The pushforward $f_{*}\left(D_{1}\right)$ is the involutive distribution

$$
\begin{aligned}
& \Delta_{2}=\operatorname{span}\left\{-3 \partial_{x^{2,+}}+\partial_{x^{4,+}}, \frac{x^{1,+}}{x^{3,+}+1} \partial_{x^{1,+}}-\partial_{x^{3,+}},\right. \\
& \left.\frac{2 x^{1,+}}{x^{3,+}+1} \partial_{x^{1,+}}-2 \partial_{x^{3,+}}-\partial_{x^{4,+}}\right\}
\end{aligned}
$$


with $\operatorname{dim}\left(\Delta_{2}\right)=\operatorname{dim}\left(D_{1}\right)=3$. In the third step, we have to find the largest projectable subdistribution of

$$
\begin{aligned}
E_{2}=\operatorname{span}\{ & -3 \partial_{x^{2}}+\partial_{x^{4}}, \frac{x^{1}}{x^{3}+1} \partial_{x^{1}}-\partial_{x^{3}}, \\
& \left.\frac{2 x^{1}}{x^{3}+1} \partial_{x^{1}}-2 \partial_{x^{3}}-\partial_{x^{4}}, \partial_{u^{1}}, \partial_{u^{2}}\right\} .
\end{aligned}
$$

In adapted coordinates (37), it can be verified that again the complete distribution is projectable, i.e., $D_{2}=E_{2}$. The pushforward $f_{*}\left(D_{2}\right)$ is the involutive distribution

$$
\Delta_{3}=\operatorname{span}\left\{\partial_{x^{1,+}}, \partial_{x^{2,+}}, \partial_{x^{3,+}}, \partial_{x^{4,+}}\right\}
$$

Here we have $\operatorname{dim}\left(\Delta_{3}\right)<\operatorname{dim}\left(D_{2}\right)=5$. However, because of $\operatorname{dim}\left(\Delta_{3}\right)=n=4$ we can stop, and according to Theorem 4 the system (36) is flat. It is important to emphasize that all these computations require only the solution of algebraic equations, and can be performed efficiently with a computer algebra program.

Now let us calculate a flat output. For this purpose, in a first step we straighten out the sequence (24) by a state transformation of the form (26) with $\bar{x}_{1}=\bar{x}_{1}^{1}, \bar{x}_{2}=\left(\bar{x}_{2}^{1}, \bar{x}_{2}^{2}\right)$, $\bar{x}_{3}=\bar{x}_{3}^{1}$ and $\operatorname{dim}\left(\bar{x}_{\text {rest }}\right)=0$. With

$$
\begin{aligned}
\bar{x}_{1}^{1}=x^{4}, \quad \bar{x}_{2}^{1}=x^{2}+3 x^{4}, \quad \bar{x}_{3}^{1}=x^{1}\left(x^{3}+1\right) \\
\bar{x}_{2}^{2}=x^{3}
\end{aligned}
$$

we get

$$
\begin{aligned}
\Delta_{1} & =\operatorname{span}\left\{\partial_{\bar{x}_{1}^{1,+}}\right\} \\
\Delta_{2} & =\operatorname{span}\left\{\partial_{\bar{x}_{1}^{1,+}}, \partial_{\bar{x}_{2}^{1,+}}, \partial_{\bar{x}_{2}^{2,+}}\right\} \\
\Delta_{3} & =\operatorname{span}\left\{\partial_{\bar{x}_{1}^{1,+}}, \partial_{\bar{x}_{2}^{1,+}}, \partial_{\bar{x}_{2}^{2,+}}, \partial_{\bar{x}_{3}^{1,+}}\right\},
\end{aligned}
$$

and the transformed system 27 reads

$$
\begin{aligned}
& \bar{x}_{3}^{1,+}=\bar{x}_{2}^{1}+\bar{x}_{2}^{2} \\
& \bar{x}_{2}^{1,+}=\bar{x}_{1}^{1}+\bar{x}_{3}^{1}\left(u^{1}+2 u^{2}\right) \\
& \bar{x}_{2}^{2,+}=u^{1}+2 u^{2} \\
& \bar{x}_{1}^{1,+}=\bar{x}_{3}^{1}+u^{2} .
\end{aligned}
$$

In a second step, we combine the coordinate transformations on $\mathcal{X} \times \mathcal{U}$ that are constructed in the proof of Theorem 4 and obtain a coordinate transformation

$$
\begin{array}{lll}
\bar{x}_{3}^{1}=y_{3}^{1} & u^{1}=\hat{z}_{1}^{2}-2 \hat{z}_{0}^{1} \\
\bar{x}_{2}^{1}=y_{2}^{1} & u^{2}=\hat{z}_{0}^{1} \\
\bar{x}_{2}^{2}=\hat{z}_{2}^{1}-y_{2}^{1} & & \\
\bar{x}_{1}^{1}=\hat{z}_{1}^{1} & &
\end{array}
$$

that straightens out the sequence (23) according to

$$
\begin{aligned}
& D_{0}=\operatorname{span}\left\{\partial_{\hat{z}_{0}^{1}}\right\} \\
& D_{1}=\operatorname{span}\left\{\partial_{\hat{z}_{0}^{1}}, \partial_{\hat{z}_{1}^{1}}, \partial_{\hat{z}_{1}^{2}}\right\} \\
& D_{2}=\operatorname{span}\left\{\partial_{\hat{z}_{0}^{1}}, \partial_{\hat{z}_{1}^{1}}, \partial_{\hat{z}_{1}^{2}}, \partial_{y_{2}^{1}}, \partial_{\hat{z}_{2}^{1}}\right\}
\end{aligned}
$$

Applying the transformation (39) and its shifted version to the right-hand side and the left-hand side of the system equations
(38) results in a structurally flat implicit triangular form

$$
\begin{array}{ll}
\Xi_{3}: & y_{3}^{1,+}-\hat{z}_{2}^{1}=0 \\
\Xi_{2}: & y_{2}^{1,+}-y_{3}^{1} \hat{z}_{1}^{2}-\hat{z}_{1}^{1}=0 \\
\Xi_{1}^{1,+}: & \hat{z}_{1}^{1,+}-y_{2}^{1,+}-\hat{z}_{1}^{2}=0
\end{array}
$$

as it is discussed in [25] (see [26] for a continuous-time counterpart). This triangular representation allows to read off a flat output $y=\left(y_{3}^{1}, y_{2}^{1}\right)$ and to systematically determine the parameterization of the other system variables by evaluating the equations (40) from top to bottom. From the topmost block $\Xi_{3}$ we get the parameterization of the variable $\hat{z}_{2}^{1}$, from $\Xi_{2}$ we get $\hat{z}_{1}^{1}$ and $\hat{z}_{1}^{2}$, and from $\Xi_{1}$ we finally get $\hat{z}_{0}^{1}$. In original coordinates, the flat output is given by $y=\left(x^{1}\left(x^{3}+1\right), x^{2}+3 x^{4}\right)$.

\section{REFERENCES}

[1] M. Fliess, J. Lévine, P. Martin, and P. Rouchon, "Sur les systèmes non linéaires différentiellement plats," Comptes rendus de l'Académie des sciences. Série I, Mathématique, vol. 315, pp. 619-624, 1992.

[2] — "Flatness and defect of non-linear systems: introductory theory and examples," Int. J. Control, vol. 61, no. 6, pp. 1327-1361, 1995.

[3] —, "A Lie-Bäcklund approach to equivalence and flatness of nonlinear systems," IEEE Trans. Autom. Control, vol. 44, no. 5, pp. 922-937, 1999.

[4] H. Sira-Ramirez and S. Agrawal, Differentially Flat Systems. New York: Marcel Dekker, 2004.

[5] A. Kaldmäe and Ü. Kotta, "On flatness of discrete-time nonlinear systems," in Proc. 9th IFAC Symposium on Nonlinear Control Systems (NOLCOS), 2013, pp. 588-593.

[6] B. Kolar, A. Kaldmäe, M. Schöberl, Ü. Kotta, and K. Schlacher, "Construction of flat outputs of nonlinear discrete-time systems in a geometric and an algebraic framework," IFAC-PapersOnLine, vol. 49, no. 18, pp. 796-801, 2016.

[7] E. Aranda-Bricaire and C. Moog, "Linearization of discrete-time systems by exogenous dynamic feedback," Automatica, vol. 44, no. 7, pp. 1707$1717,2008$.

[8] P. Guillot and G. Millérioux, "Flatness and submersivity of discrete-time dynamical systems," IEEE Control Syst. Lett., vol. 4, no. 2, pp. 337-342, 2020.

[9] G. Millérioux and R. Jungers, "Characterization of flat outputs of switched linear discrete-time systems: Algebraic condition and algorithm," Syst. Control Lett., vol. 154, 2021.

[10] E. Aranda-Bricaire, Ü. Kotta, and C. Moog, "Linearization of discretetime systems," SIAM J. Control Optim., vol. 34, no. 6, pp. 1999-2023, 1996.

[11] C. Califano, S. Monaco, and D. Normand-Cyrot, "On the problem of feedback linearization," Syst. Control Lett., vol. 36, no. 1, pp. 61-67, 1999.

[12] — "Non-linear non-interacting control with stability in discrete-time: A geometric framework," Int. J. Control, vol. 75, no. 1, pp. 11-22, 2002.

[13] J. Grizzle, "Feedback linearization of discrete-time systems," in Analysis and Optimization of Systems, ser. Lecture Notes in Control and Information Sciences, A. Bensoussan and J. Lions, Eds. Berlin: Springer, 1986, vol. 83, pp. 273-281.

[14] B. Jakubczyk, "Feedback linearization of discrete-time systems," Syst. Control Lett., vol. 9, no. 5, pp. 411-416, 1987.

[15] B. Jakubczyk and W. Respondek, "On linearization of control systems," Bull. Acad. Polonaise Sci. Ser. Sci. Math., vol. 28, pp. 517-522, 1980.

[16] A. van der Schaft, "Linearization and input-output decoupling for general nonlinear systems," Syst. Control Lett., vol. 5, pp. 27-33, 1984.

[17] K. Sato, "On an algorithm for checking whether or not a nonlinear discrete-time system is difference flat," in Proc. 20th International Symposium on Mathematical Theory of Networks and Systems (MTNS), 2012.

${ }^{4}$ The coordinate transformations on $\mathcal{X} \times \mathcal{U}$ constructed in the proof of Theorem 4 can be interpreted as input transformations for the corresponding subsystems. However, with regard to the complete system, they do not necessarily preserve the separation into state- and input variables. Thus, the transformation can yield in general an implicit system representation. For the system 40, however, by rearranging the equations of the block $\Xi_{2}$ also an explicit triangular form would be possible. 
[18] J. Lévine, "On necessary and sufficient conditions for differential flatness," Appl. Algebra Eng. Commun. Comput., vol. 22, no. 1, pp. 47-90, 2011.

[19] B. Kolar, M. Schöberl, and J. Diwold, "Differential-geometric decomposition of flat nonlinear discrete-time systems," Automatica, vol. 132, 2021.

[20] K. Schlacher, "Flat sampled data systems, an algorithmic approach," arXiv e-prints, 2019, arXiv:1909.00555 [math.DS].

[21] J. Grizzle, "A linear algebraic framework for the analysis of discretetime nonlinear systems," SIAM J. Control Optim., vol. 31, no. 4, pp. 1026-1044, 1993

[22] Ü. Kotta, Inversion Method in the Discrete-time Nonlinear Control Systems Synthesis Problems, ser. Lecture Notes in Control and Information Sciences. Berlin: Springer, 1995, vol. 205.

[23] H. Nijmeijer and A. van der Schaft, Nonlinear Dynamical Control Systems. New York: Springer, 1990.

[24] W. Boothby, An Introduction to Differentiable Manifolds and Riemannian Geometry, 2nd ed. Orlando: Academic Press, 1986.

[25] B. Kolar, M. Schöberl, and K. Schlacher, "A decomposition procedure for the construction of flat outputs of discrete-time nonlinear control systems," in Proc. 22nd International Symposium on Mathematical Theory of Networks and Systems (MTNS), 2016, pp. 775-782.

[26] M. Schöberl and K. Schlacher, "On an implicit triangular decomposition of nonlinear control systems that are 1-flat - a constructive approach," Automatica, vol. 50, pp. 1649-1655, 2014 